\title{
Screening for tuberculosis among high- risk groups attending London emergency departments: a prospective observational study
}

\begin{abstract}
To the Editor:
Most tuberculosis (TB) cases in low-incidence settings are thought to be due to reactivation of latent TB infection (LTBI) in high-risk populations [1-3]. Assessment of patients at emergency departments (EDs) is a potential opportunity to achieve early TB diagnosis, and interrupt transmission. An earlier study in London found that 39\% of patients diagnosed with TB had attended an ED in the preceding 6 months [4]. Of these, $76 \%$ had a chest radiograph performed, of which $86 \%$ and $40 \%$ were abnormal in cases of pulmonary and extrapulmonary TB, respectively. Attendance at EDs provides an opportunity to identify individuals with LTBI, who may be at risk for progression to active disease and unlikely to engage with healthcare services via other routes.
\end{abstract}

Between July 2013 and May 2017, we recruited individuals over the age of 16 years, who were recent entrants from, or prolonged travellers to, high TB incidence countries, or people with a history of homelessness, imprisonment or problem drug use attending EDs at seven London hospitals. We investigated the yield of interferon- $\gamma$ release assay (IGRA) and TB disease screening among eligible ED attendees, regardless of their reason for ED attendance. Participants were tested with either the QuantiFERON Gold-in-Tube (Qiagen, Hilden, Germany), or QuantiFERON-TB Plus. A subset of participants was screened for TB disease; symptomatic individuals ( $\geqslant 2$ week history of cough or fever, accompanied by haemoptysis, drenching night sweats, or unexplained weight loss) underwent chest radiograph, sputum Xpert $\mathrm{MTB} / \mathrm{RIF}$ testing and subsequent referral for further evaluation and management [5]. Those screened with IGRA were followed-up through data linkage to national TB surveillance notifications to identify subsequent active TB notification [6]. This study was approved by the Stanmore National Health Service Research Ethics Committee (14/LO/2160) and registered on ClinicalTrials.gov (NCT02512484; full study protocol available at the institutional website [7]).

Descriptive analyses were performed to assess the yield of screening and incidence rates of TB disease among those tested by IGRA. Logistic regression models were used to examine factors associated with IGRA positivity. The final multivariable model included a priori variables (age, sex, presence of social risk factors, history of TB contact, ethnicity and country of birth), and variables found to be significant in univariable analyses $(\mathrm{p}<0.2)$.

A total of 1407 participants were recruited to the IGRA screening study, of whom 241 (17.1\%) had a history of substance use disorders, homelessness or imprisonment, while the remainder were migrants from high TB burden countries. Of the 1407 participants, 642 (45.6\%) were female, and the majority (1010; 70.8\%) were over 35 years of age (median 45 years). Among those recruited due to recent migration from, or travel to, a high TB incidence country, most participants (736/1166; 63.1\%) were South Asian, while the largest ethnic group among those with social risk factors was white $(100 / 241 ; 41.5 \%)$. Almost one fifth $(258 / 1407 ; 18.2 \%)$ of participants reported previous contact with a TB case. Diabetes was common, affecting 239/1407 (17.0\%). Of the 1407 participants, $109(7.7 \%)$ were not registered with a general practitioner; this was more common among those with social risk factors $(35 / 241 ; 14.5 \%)$ when compared with the migrant group $(74 / 1166 ; 6.3 \%)$.

$@$ ERSpublications

LTBI screening among high-risk groups at EDs could be implemented to identify those at risk of progression to TB disease. Large-scale studies are required to investigate effective TB disease screening strategies in EDs. https://bit.ly/3bTkoOn

Cite this article as: Gupta RK, Lule SA, Krutikov M, et al. Screening for tuberculosis among high-risk groups attending London emergency departments: a prospective observational study. Eur Respir J 2021; 57: 2003831 [https://doi.org/10.1183/13993003.03831-2020]. 
IGRA results were available for 1232 participants, of which 34 (2.4\%) were indeterminate. A total of 256/ 1198 (21.4\%) participants with valid available results were IGRA positive. The prevalence of IGRA positivity was $24 \%$ among migrants and $19 \%$ among those with social risk factors (table 1 ). In a multivariable logistic regression model, only male sex (OR 1.38, 95\% CI 1.01-1.87; $\mathrm{p}=0.041$ ), age $>35$ years (OR 1.67, 95\% CI 1.12-2.55; $\mathrm{p}=0.012$ ) and non-UK country of birth (OR 6.32, 95\% CI 2.99-15.6; $\mathrm{p}<0.001$ ) were independently associated with IGRA positivity.

Participants screened by IGRA were followed up for a median of 381 days (interquartile range 303605 days), via linkage to national TB surveillance records until 31 December 2017. Of the 256 with a positive IGRA, five were notified with TB disease during follow-up, giving a TB incidence rate among those with a positive IGRA of $1476.4 / 100000$ person-years (95\% CI 529.4-3173.2). All five TB cases had extrapulmonary disease (lymph node (notified 5 days after recruitment), spinal (35 days), disseminated (52 days), genito-urinary (162 days) and intra-abdominal (412 days)), and none reported recent TB contact at study recruitment. Median quantitative interferon- $\gamma$ responses to Mycobacterium tuberculosis antigens were $6.02 \mathrm{IU} \cdot \mathrm{mL}^{-1}$ (range 0.62 to $>10 \mathrm{IU} \cdot \mathrm{mL}^{-1}$ ) among the five progressors to TB disease, compared to $2.57 \mathrm{IU} \cdot \mathrm{mL}^{-1}\left(0.35\right.$ to $\left.>10 \mathrm{IU} \cdot \mathrm{mL}^{-1}\right)$ among non-progressors. No TB cases were notified among participants who had negative or indeterminate IGRAs.

Of the 513 participants screened in the active TB study, only 14 (2.7\%) were symptomatic. Of these, 13 had a chest radiograph, with eight providing an adequate sputum sample for Xpert MTB/RIF testing. None of these patients were diagnosed with TB during the study.

TABLE 1 Factors associated with interferon- $\gamma$ release assay (IGRA) positivity in univariable and multivariable logistic regression models

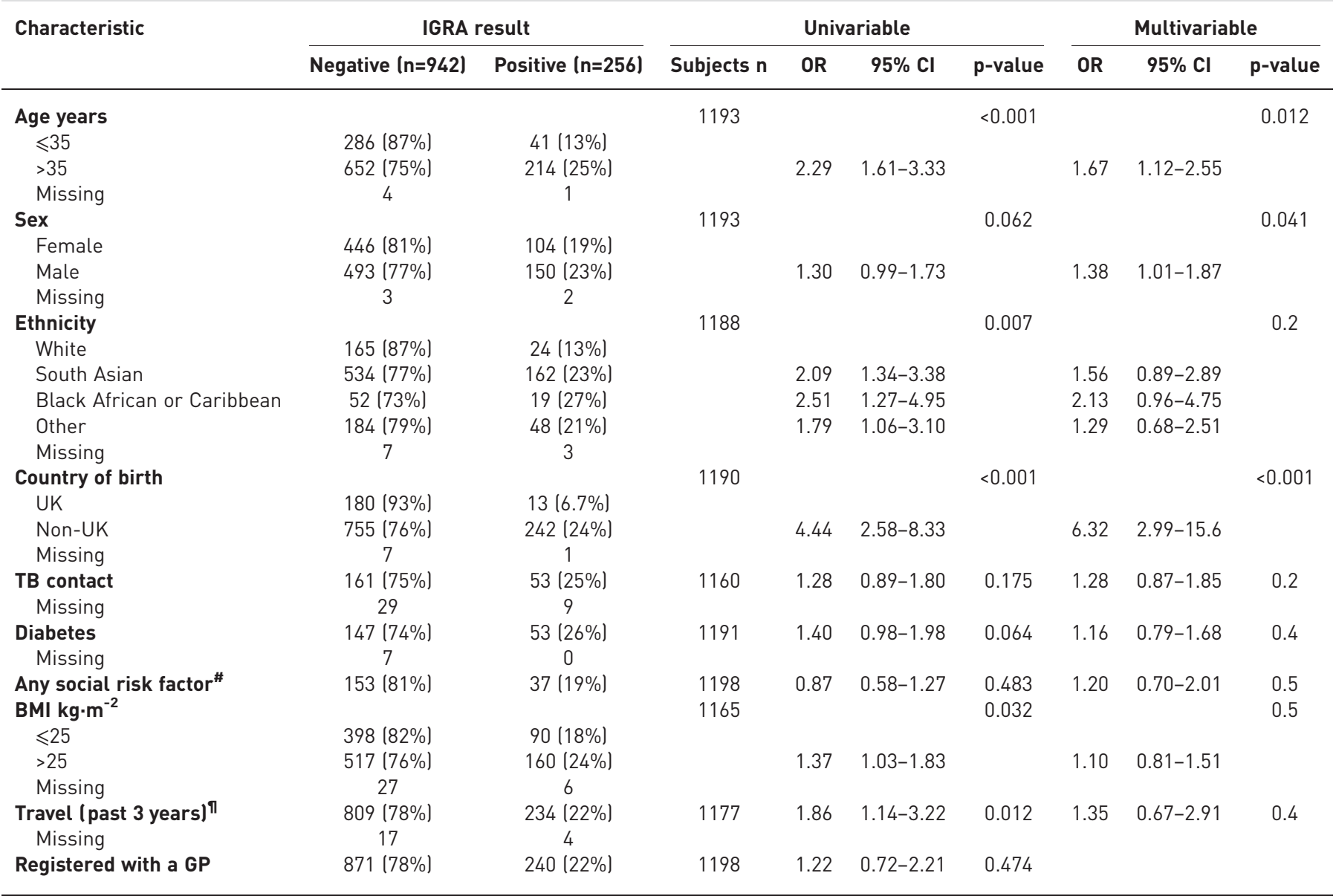

Data shown for participants with valid IGRA results $(n=1198) . n=1027$ in multivariable model. Variables were included in the model if considered of clinical importance a priori, or if significant in univariate analysis ( $p<0.2)$. Percentages reflect row percentages. TB: tuberculosis; BMI: body mass index; GP: general practitioner. \#: includes history of homelessness, imprisonment or harmful drug use; ${ }^{\natural}$ : indicates any travel to a high TB incidence country in the past 3 years. 
Previous studies have retrospectively reviewed ED presentations among notified TB cases in low-incidence settings [4, 8-11], and examined the yield of TB screening in high-incidence countries [12, 13]. A study in the USA also investigated the yield of LTBI screening among risk groups attending EDs using the tuberculin skin test (TST) [14]. Notably, nearly half of the participants in the US study did not return to have the TST read. Our study has demonstrated the feasibility of prospective IGRA testing among high-risk groups attending EDs in London. Valid IGRA results were available for $85 \%$ of participants, which is comparable to other IGRA evaluations $[15,16]$. Linkage to national TB surveillance records provided a previously validated mechanism to identify participants screened by IGRA who subsequently progressed to TB disease [6], with median follow-up longer than 1 year.

Major study limitations were that patients with positive IGRA results were not routinely linked to TB services for evaluation for TB disease and consideration of LTBI treatment, since LTBI screening among risk groups attending EDs was not part of national policy at the time. Thus, we are unable to estimate the proportions of IGRA-positive participants who would have started and completed preventative therapy if referred to TB services following detection. This remains a key knowledge gap, since effectiveness (and cost-effectiveness) of screening is dependent upon completion of LTBI treatment for these individuals, to reduce risk of $\mathrm{TB}$ disease. In addition, although the vast majority of participants in the study were registered with general practitioners, it is unclear how engaged they were with these services, limiting our ability to assess precisely how well ED screening could complement the primary care screening programme. Data were not available on individuals who attended EDs and were screened for eligibility but not recruited to the study.

In the $\mathrm{TB}$ disease screening study arm, we proactively screened for pulmonary $\mathrm{TB}$ disease using a symptom screen followed by chest radiograph and sputum testing. Consequently, pulmonary TB cases without typical symptoms and extrapulmonary cases may have been missed. Moreover, our sample size of 513 participants screened for TB symptoms may have been too small to detect active cases; we therefore could not evaluate risk factors for active TB or cost-effectiveness of TB disease screening.

In summary, our study suggests that ED IGRA screening among TB risk groups could be implemented to identify individuals at risk of TB who may be difficult to engage via other screening approaches. Such screening must be supported by a dedicated protocol that detects individuals at higher risk of TB for screening, and resources to facilitate onward referral of those with a positive IGRA to local TB services. In contrast, this study suggests that resource-intensive, symptom-based active TB screening in EDs is unlikely to be worthwhile as the yield is likely to be too low to justify the resource. However, other high-throughput screening models such as automated, routine chest radiograph review in EDs may be evaluated in future research.

Rishi K. Gupta $\oplus^{1}$, Swaib A. Lule ${ }^{1}$, Maria Krutikov $\oplus^{1}$, Lara Gosce $\odot^{1}$, Nathan Green ${ }^{2}$, Jo Southern ${ }^{3}$, Ambreen Imran $\oplus^{4}$, Robert W. Aldridge $\odot^{5}$, Heinke Kunst ${ }^{6}$, Marc Lipman $\oplus^{4,7}$, William Lynn ${ }^{8}$, Helen Burgess ${ }^{9}$, Asif Rahman $^{10}$, Dee Menezes ${ }^{5}$, Ananna Rahman ${ }^{6}$, Simon Tiberi ${ }^{6,11}$, Peter J. White ${ }^{2,12}$ and Ibrahim Abubakar ${ }^{1}$

${ }^{1}$ Institute for Global Health, University College London, London, UK. ${ }^{2}$ MRC Centre for Global Infectious Disease Analysis and NIHR Health Protection Research Unit in Modelling and Health Economics, Imperial College London, London, UK. ${ }^{3}$ TB Unit, Public Health England, Colindale, London, UK. ${ }^{4}$ Royal Free London NHS Foundation Trust, London, UK. ${ }^{5}$ Centre for Public Health Data Science, Institute of Health Informatics, University College London, London, UK. ${ }^{6}$ Blizard Institute, Queen Mary University of London, London, UK. ${ }^{7}$ UCL-TB and UCL Respiratory, University College London, London, UK. ${ }^{8}$ London North West University NHS Trust, London, UK. ${ }^{9}$ West Middlesex University Hospital, Chelsea and Westminster NHS Foundation Trust, London, UK. ${ }^{10}$ Imperial College London NHS Trust, London, UK. ${ }^{11}$ Division of Infection, Barts Health NHS Trust, London, UK. ${ }^{12}$ Modelling and Economics Unit, National Infection Service, Public Health England, London, UK.

Correspondence: Ibrahim Abubakar, Institute for Global Health, University College London, 30 Guilford Street, London, UK. E-mail: i.abubakar@ucl.ac.uk

This study is registered at ClinicalTrials.gov (NCT02512484). The authors agree to share the data on reasonable request.

Received: 13 Oct 2020 | Accepted: 23 Feb 2021

Acknowledgements: We are grateful to all the ED staff who were willing to allow us into their busy departments to investigate the value of TB screening, to the patients who agreed to participate in this study during their visit to the ED for unrelated reasons, the laboratory staff who provided an excellent testing service, and local TB specialist nursing teams who facilitated participant follow-up.

Author contributions: R.K. Gupta and I. Abubakar wrote the first draft of the manuscript. R.K. Gupta, M. Krutikov, S.A. Lule and I. Abubakar were responsible for the critical review of the draft. R.K. Gupta, L. Gosce and N. Green performed the analysis with oversight from P.J. White and I. Abubakar. I. Abubakar conceived and designed the study, with support from J. Southern, R.W. Aldridge and Asif Rahman. I. Abubakar and J. Southern led recruitment of participants with all site investigators (including M. Lipman, H. Kunst, W. Lynn, H. Burgess, S. Tiberi and Asif 
Rahman). A. Imran and Ananna Rahman contributed to participant recruitment and follow-up. All other authors contributed to study design, analysis or interpretation. All authors have seen and agreed on the final submitted version of the manuscript. The corresponding author affirms that the manuscript is an honest, accurate, and transparent account of the study being reported and that no important aspects of the study have been omitted.

Conflict of interest: R.K. Gupta has nothing to disclose. S.A. Lule has nothing to disclose. M. Krutikov has nothing to disclose. L. Gosce has nothing to disclose. N. Green has nothing to disclose. J. Southern has nothing to disclose. A. Imran has nothing to disclose. R.W. Aldridge has nothing to disclose. H. Kunst has nothing to disclose. M. Lipman has nothing to disclose. W. Lynn has nothing to disclose. H. Burgess has nothing to disclose. A. Rahman has nothing to disclose. D. Menezes has nothing to disclose. A. Rahman has nothing to disclose. S. Tiberi has nothing to disclose. P.J. White reports grants from Medical Research Council and National Institute for Health Research, during the conduct of the study; grants from National Institute for Health Research, outside the submitted work. I. Abubakar reports grants from UK Department of Health, during the conduct of the study.

Support statement: This work was supported by the National Institute for Health Research (NIHR) through Policy Research Programme (reference 015/0307), and personal awards (DRF-2018-11-ST2-004 to R.K. Gupta; 206602/Z/17/Z to R.W. Aldridge; SRF-2011-04-001 to I. Abubakar; NF-SI-0616-10037 to I. Abubakar). P.J. White, I. Abubakar and N. Green received funding from NIHR Health Technology Assessment (NIHR127459). S.A. Lule was supported by the PANDORA-ID-NET Consortium (EDCTP Reg/Grant RIA2016E-1609) funded by the European and Developing Countries Clinical Trials Partnership (EDCTP2) programme under the Horizon 2020, the European Union's Framework Programme for Research and Innovation. P.J. White and N. Green acknowledge funding from the UK National Institute for Health Research (NIHR) Health Protection Research Unit (HPRU) in Modelling Methodology at Imperial College London, in partnership with Public Health England (HPRU-2012-10080). P.J. White also thanks the NIHR HPRU in Modelling and Health Economics, a partnership between PHE, Imperial College London and LSHTM, for funding (NIHR200908). P.J. White also acknowledges support from the Medical Research Council (MRC) Centre for Global Infectious Disease Analysis (MR/R015600/1); this award is jointly funded by the MRC and the UK Foreign, Commonwealth and Development Office (FCDO) under the MRC/FCDO Concordat agreement and is also part of the European and Developing Countries Clinical Trials Partnership 2 (EDCTP2) programme supported by the EU. This paper presents independent research supported by the NIHR. The views expressed are those of the author(s) and not necessarily those of the Department of Health and Social Care, FCDO, MRC, NHS, NIHR, or Public Health England. Funding information for this article has been deposited with the Crossref Funder Registry.

\section{References}

1 The National Institute for Health and Care Excellence. NICE Guidance and Guidelines: Tuberculosis. London, NICE, 2016.

2 Public Health England. Collaborative Tuberculosis Strategy for England. London, Public Health England, 2015.

3 Aldridge RW, Zenner D, White PJ, et al. Tuberculosis in migrants moving from high-incidence to low-incidence countries: a population-based cohort study of 519955 migrants screened before entry to England, Wales, and Northern Ireland. Lancet 2016; 388: 2510-2518.

4 Appleton SC, Connell DW, Singanayagam A, et al. Evaluation of prediagnosis emergency department presentations in patients with active tuberculosis: the role of chest radiography, risk factors and symptoms. $B M$ J Open Respir Res 2017; 4: e000154.

5 World Health Organization. Using the Xpert MTB/RIF assay to detect pulmonary and extrapulmonary tuberculosis and rifampicin resistance in adults and children. Expert group meeting report. Geneva, World Health Organization, 2013.

6 Aldridge RW, Shaji K, Hayward AC, et al. Accuracy of probabilistic linkage using the enhanced matching system for public health and epidemiological studies. PLoS One 2015; 10: e0136179.

7 University College London. Improving the Detection of Active Tuberculosis in Accident and Emergency Departments. Date last updated: 26 Feb 2016. Date last accessed: 5 Dec 2020. www.ucl.ac.uk/global-health/sites/ global-health/files/ace_active_tb_study_protocol_version_3.0_dated_26.02.16.pdf

8 Geyer BC, Godwin P, Powell TJ, et al. Patient factors associated with failure to diagnose tuberculosis in the emergency department. J Emerg Med 2013; 45: 658-665.

9 Sokolove PE, Rossman L, Cohen SH. The emergency department presentation of patients with active pulmonary tuberculosis. Acad Emerg Med 2000; 7: 1056-1060.

10 Lad TS, Packe GE. Tuberculosis: a missed opportunity for early diagnosis at the front line? Emerg Med J 2014; 31: 942-943.

11 Smith A, Miller RF, Story A, et al. A\&E department: a missed opportunity for diagnosis of TB? Thorax 2006; 61: 364-365.

12 Silva DR, Müller AM, Tomasini KS, et al. Active case finding of tuberculosis (TB) in an emergency room in a region with high prevalence of TB in Brazil. PLoS One 2014; 9: e107576.

13 Ticona E, Huaman MA, Huaroto LM, et al. Tuberculosis screening using ability to provide sputum in an endemic emergency department. Eur Respir J 2016; 47: 330-333.

14 Kirsch TD, Chanmugam A, Keyl P, et al. Feasibility of an emergency department-based tuberculosis counseling and screening program. Acad Emerg Med 1999; 6: 224-231.

15 Gupta RK, Kunst H, Lipman M, et al. Evaluation of QuantiFERON-TB gold plus for predicting incident tuberculosis among recent contacts: a prospective cohort study. Ann Am Thorac Soc 2020; 17: 646-650.

16 Abubakar I, Drobniewski F, Southern J, et al. Prognostic value of interferon-gamma release assays and tuberculin skin test in predicting the development of active tuberculosis (UK PREDICT TB): a prospective cohort study. Lancet Infect Dis 2018; 18: 1077-1087. 